UCRL-JC-117506

PREPRINT

\title{
Electromechanical Battery Program at the Lawrence Livermore National Laboratory
}

\author{
Richard F. Post, Donald A. Bender \\ and Bernard T. Merritt
}

This paper was prepared for submittal to

Intersociety Energy Conversion Engineering Conference

Monterey, $\mathrm{CA}$

August 7-12, 1994

May 31, 1994

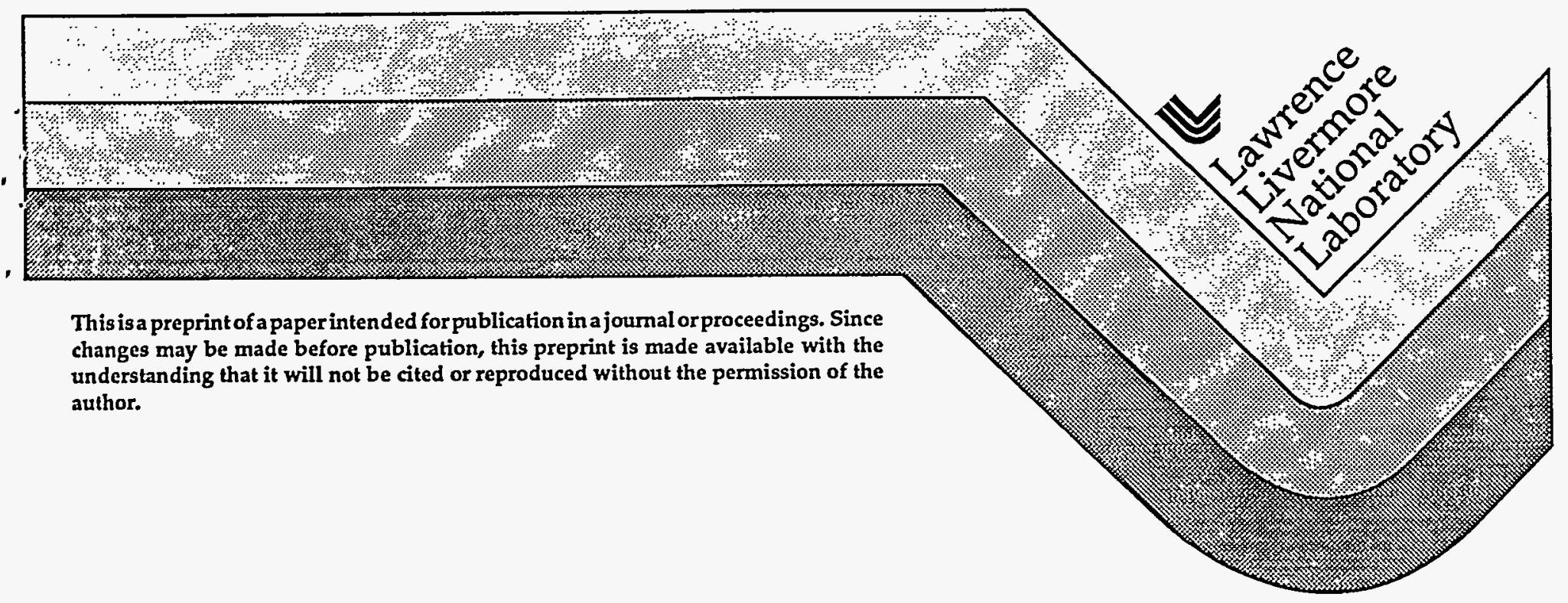




\section{DISCLAIMER}

This report was prepared as an account of work sponsored by an agency of the United States Government. Neither the United States Government nor any agency thereof, nor any of their employees, make any warranty, express or implied, or assumes any legal liability or responsibility for the accuracy, completeness, or usefulness of any information, apparatus, product, or process disclosed, or represents that its use would not infringe privately owned rights. Reference herein to any specific commercial product, process, or service by trade name, trademark, manufacturer, or otherwise does not necessarily constitute or imply its endorsement, recommendation, or favoring by the United States Government or any agency thereof. The views and opinions of authors expressed herein do not necessarily state or reflect those of the United States Government or any agency thereof. 


\section{DISCLAIMER}

Portions of this document may be illegible in electronic image products. Images are produced from the best available original document. 


\title{
ELECTROMECHANICAL BATTERY PROGRAM AT THE LAWRENCE LIVERMORE NATIONAL LABORATORY
}

\author{
Richard F. Post, Donald A. Bender, and Bernard T. Merritt \\ Lawrence Livermore National Laboratory, P.O. Box 808, L-640, Livermore, CA 94550
}

\begin{abstract}
New materials and new design concepts are being incorporated in a new approach to an old idea - flywheel energy storage - to create an important alternative to the electrochemical storage battery for use in electric vehicles or for stationary applications, such as computer back-up power or utility load-leveling. We visualize such EMBs (electro-mechanical batteries) as being modular in character, with small (1-5 kWh) modules being used for power-conditioning and for vehicular use, and paralleled $25 \mathrm{kWh}$ modules being used for bulk storage, i.e., load-leveling, applications. In a funded program at the Laboratory two fractional $\mathrm{kWh}, 200 \mathrm{~kW}$ (design peak power) modules have been constructed and subjected to shake-down testing. Their design for high peak power was prompted by awareness of a particular commercial need, as a component in a power-line conditioning device. In addition to such stationary applications, the high power capability of our EMB designs makes them attractive for use in hybrid-electric vehicles. Important elements of the LLNL program include the development of passive magnetic bearings and the application of new high-efficiency permanent magnet arrays to an ironless generator/motor. Use of these particular elements, together with a multi-ring design for the flywheel rotor, is particularly conducive to a systemic approach to the design of an EMB module. These particular design developments have been motivated by the economic issues of simplification, lowered cost, and extended maintenance-free service life.
\end{abstract}

\section{I) INTRODUCTION}

At the Lawrence Livermore National Laboratory we have an on-going program to develop modular electromechanical batteries (EMBs) based on new materials and novel design concepts. We believe that these EMBs have many applications, both stationary and mobile. For example, we visualize using $1 \mathrm{kWh}$ modules for electric and hybrid-electric automobiles (larger modules for buses and locomotives), and 2 to $25 \mathrm{kWh}$ modules for stationary applications such as power conditioning, load shifting and distributed storage.

An EMB, as suggested by its name, is the mechanical equivalent of an electrochemical storage battery. As visualized by us an EMB is a modular system consisting of a highspeed fiber-composite rotor, supported by a magnetic bearing system, integrated with a special ironless generator/motor, and housed in a sealed, evacuated, enclosure. Electrical

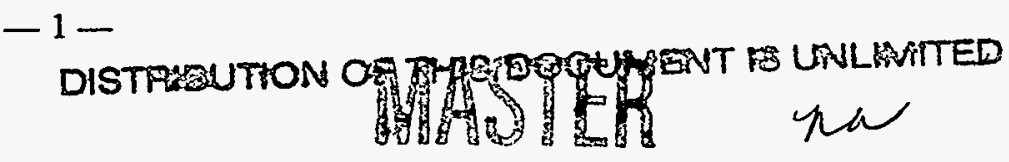


energy is coupled into and out of the module inductively, through a re-entrant vacuum barrier. These "systems-oriented" design concepts, plus the employment of the latest super-strength carbon fibers in the rotor and high-field permanent magnet material in the generator/motor and in the magnetic bearings are essential to achieving the predicted high performance.

The performance characteristics of the EMB modules that we are developing include: very high specific power, 10 or more $\mathrm{kW} / \mathrm{kg}$, and very high energy recovery efficiencies $(\mathrm{kWh}$ out vs $\mathrm{kWh}$ in), up to $95 \%$ compared to a typical 60 to $70 \%$ for electrochemical cells. In addition, we believe that our EMBs should have a very long and maintenance-free service lifetime, ten years or more, this even under virtually unlimited numbers of deep-discharge cycles. These characteristics set the EMB apart from any known electrochemical cell.

Because the rotor of the EMB rotates in vacuo at speeds as high as 200,000 RPM (for small modules), the only practical way to support it against the force of gravity, minimize frictional drag, and achieve long service life is to use a magnetic suspension/bearing system. At the Laboratory we have under development some new concepts for magnetic bearings that promise to be much simpler and less expensive than the servo-controlled bearings that are now in use in specialty applications. Our developmental bearings are of the "passive" type, employing permanent magnets to supply the levitating forces, and rotordynamic effects to achieve stability.

Until recently financial support for our EMB program has come from the Laboratory's LDRD (Laboratory Directed Research and Development) program, a precursor to obtaining funding from outside sources. Such outside support has recently been obtained, in the form of a CRADA (Cooperative Research and Development Agreement), with two companies: Trinity Flywheel Batteries, Inc. of San Francisco, and the Westinghouse Electric Corporation. This report describes the progress in our program up to the time of initiation of that CRADA.

Our progress during the year preceding that date included tests of all components of an EMB (bearings, generator, vacuum in a sealed-off chamber, etc.) and the construction of two operable modules. One module, designed to store about $1 \mathrm{kWh}$ and deliver up to $200 \mathrm{~kW}$ in its short-discharge mode of operation, was spun up in initial testing, prior to moving to a test cell. In "bench-top", reduced-speed, tests it delivered $20 \mathrm{~kW}$ output (single-phase) at 20,000 RPM with a measured two-way energy recovery efficiency (at the output leads) of $92 \%$. A speed of 20,000 RPM for that module corresponds to $33 \%$ of its design peak speed of 60,000 RPM. With increase in the speed up to the operating speed 
range, and with multi-phase (3-phase) windings on the stator it is reasonable to expect output powers in excess of $100 \mathrm{~kW}$, and two-way energy recovery efficiencies of $95 \%$ or more.

\section{II) DESIGN FEATURES}

The salient design features of our version of an EMB have been described in a previous publication. 1 These features are illustrated in Fig. 1, a cutaway drawing that represents schematically a sectioned view of a small module. For use in an all-electric or a hybrid-electric auto the module would be cubical in shape, having outside dimensions of order $25 \mathrm{~cm}$ and storing approximately $1 \mathrm{kWh}$. The rotor of the module is made up of nested cylindrical shells fabricated of high-strength graphite-fiber/epoxy composite. These shells are coupled to each other by "separators". As noted earlier, the rotor is housed in a sealed, evacuated, chamber and is supported on magnetic bearings.

The innermost shell of the rotor has mounted on its inner surface a special assembly (the "Halbach Array" - described later) of high-field permanent magnet material (NdFeB). Inside of this magnet array is a re-entrant cylinder of insulating material that serves as a vacuum barrier through which the field of the rotating array is coupled to three-phase stator windings. In this way the windings are made directly accessible for cooling, either by forced air or by a liquid coolant. The entire generator/motor assembly is "ironless", a feature that leads to high efficiency and low standby losses (no hysteresis effects). Because of the high rotation speed of the rotor it also leads to a very high specific powers ( 5 to $10 \mathrm{~kW} / \mathrm{kg}$; up to two orders of magnitude greater than those typical of electrochemical cells).

Also shown in Fig. 1, albeit only schematically, are the magnetic bearing assemblies, with their permanent magnet exciters. These bearings, which will not be discussed here in detail, also benefit from the use of $\mathrm{NdFeB}$ magnet material. Only a relatively few grams of this material will be required to provide the magnetomotive force needed to support the rotor. However, the magnetic bearings represent one of the most challenging of the design problems for compact EMBs. As a consequence we have made a special effort to develop simplified versions of these bearings, one embodiment of which will be employed in EMB modules to be developed.

The chamber within which the rotor/bearing assembly contained is a sealed, evacuated, metal housing reinforced with fiber composite. This reinforcement is both to sustain vacuum-induced loads, and also to provide a first level of defense against the consequences of rotor failure. Safe containment of the debris and of the energy released upon rotor 
failure is an essential requirement for any vehicular use of EMBs. To address this issue fully will require an extensive test program. However, we believe that experience gained in the aircraft industry, where similar problems are encountered in the failure of turbo-jet engines, can be applied to help solve the problem in vehicular EMBs. As described in a NASA report, ${ }^{2}$ structures containing only a few kilograms of polymeric fiber provide containment in the event of blade failure in large jet turbines.

The module just described is one that is sized for vehicular applications. For other applications we would expect to scale up the module size somewhat. For example, for the computer-backup application and for power-conditioning applications 2 to $5 \mathrm{kWh}$ modules would be desirable, while for load-leveling and bulk-storage applications $25 \mathrm{kWh}$ units (used in a "battery-bank" mode) appear to represent nearly an optimum size, considering economic factors such as manufacturability, shipping, and installation. For the rotors of the latter units the fiber of choice would likely not be graphite fiber (presently too expensive per $\mathrm{kWh}$ stored), but instead the workhorse fiber, "E-glass", would be used, under temperature controlled conditions to minimize stress-corrosion effects.

\section{III) SPECIAL DESIGN ISSUES}

\section{A. The Rotor}

Our choice of a multi-shell configuration for the rotor of the EMB is based on both practical and economic considerations. The first consideration is a materials-related one, having to do with the mechanical properties of fiber composites, as follows: To achieve maximum strength in hoop tension, the fiber composite of which the cylindrical shells the rotor are composed is "uni-directional" in nature. That is, the shells are to be fabricated (by automated filament winding) with the filaments oriented azimuthally. As a consequence, although maximal strength against centrifugal hoop tension is achieved, it is at the sacrifice of strength in the radial direction, where the tensile strength is only that of the epoxy matrix. However, owing to the radial gradient of centrifugal forces internal radial stresses will arise within the body of the ring. Theory shows that these stresses vary roughly as the square of the radial thickness of the shells. However, by limiting the thickness of the rings to approximately $10 \%$ of their radius the internal tensile stresses can be kept well within allowable limits. We have incorporated this feature in our projected designs.

Another feature of our particular multi-shell rotor design is that of mass loading the inner rings. The effects of mass loading are threefold: First, loading increases the amount of energy stored in the rotor per unit volume, with only a modest decrease in the specific energy. Second, loading diminishes the amount by which the gap between adjacent rings grows under centrifugal stress, thus simplifying the job of designing the separators. Third, 
loading can reduce the radial tensile stress within the shells by introducing a compressional term, even including transforming those stresses to pure compression. Note that mass loading of the innermost shell of the rotor occurs naturally because of the presence of the permanent magnets of the Halbach Array.

Additional issues associated with the multi-shell rotor design are those of resonances and rotational instabilities of the rotor body itself. One such class of unstable resonance response arises from the transverse oscillations of the shells of the rotor with respect to each other. These oscillations were analyzed theoretically early on and subjected to experimental test, with close agreement between the experiments and theory. ${ }^{3}$ It was shown that instability can be avoided if the lowest mode of transverse oscillation (determined by the effective spring constants of the separators) is constrained to lie above the highest operating speed of the rotor.

The remaining class of rotational instabilities are so-called "whirl" instabilities, arising from dissipative losses (and consequent out-of-phase torques) occurring within the rotating system. The general prescription for eliminating these in a rotor supported by magnetic bearings is to incorporate compliant and/or dissipative elements in the bearing supports. ${ }^{4}$

Compliance also plays another important role in our designs. To avoid the necessity of precision balancing, which would be difficult (if not impossible) in a fiber-composite rotor of our configuration, operation is to be "super-critical". That is, by design the operating speed of the rotor lies far above the first critical speed determined by the mass of the rotor and the compliance of the suspension/bearing system. The validity of this concept for a multi-ring flywheel system was demonstrated in tests performed in the 1970 s. $^{5}$

\section{B. Vacuum-Related Issues}

It is necessary to operate the rotor in vacuo in order to avoid excessive aerodynamic losses. In our design the rotor is to be housed in an evacuated chamber that is sealed off at the time of manufacture. The degree of vacuum required for an acceptably long self-discharge time can be estimated approximately from kinetic theory. For a $1 \mathrm{kWh}$ rotor the calculated rundown time constant (from aerodynamic losses) at a residual gas pressure of $3 \times 10^{-}$ 5) Torr is 60 days.

We have performed preliminary tests, in a sealed-off chamber, that indicate that we should be able to maintain the required vacuum without the necessity for continuous pumping. The tests were performed at various temperatures from ambient to $100^{\circ} \mathrm{C}$, using a specially treated and pre-processed epoxy-graphite fiber composite structure of the same general size 
as the rotor. Figure 2 shows a pressure vs time curve for the system following seal-off, when maintained at a temperature of $50^{\circ} \mathrm{C}$ (to simulate the highest ambient temperature likely to be encountered in vehicular use). As can be seen, the pressure level remains acceptably low even when the chamber is no longer being pumped. This pressure was maintained for a period of months, before the test was terminated.

\section{The Generator/Motor}

A central feature of our version of the EMB is the generator/motor design. As mentioned previously, our design is an "ironless" one, utilizing a special array of permanent magnet bars to create a rotating dipole field. This array is one that was pioneered by Klaus Halbach of the Lawrence Berkeley Laboratory. ${ }^{6}$ Figure 3 shows the dipole version of Halbach's array, together with the calculated lines of force of the field that it produces. Note the uniformity of the interior field, and its near-cancellation outside the array. The efficiency of the Halbach array is such that using $\mathrm{NdFeB}$ material for the magnets, dipole fields of order 0.5 Torr can readily be obtained.

Both the predicted power output and the efficiency of the generator motor in our example 1 $\mathrm{kWh}$ module are remarkably high. There are no hysteresis losses in the ironless system, and since the inductance of the multi-phase windings are consequently very low, and rotation speeds are high, unusually high peak power outputs are possible, with stator copper losses that can readily be handled by conventional means (air or liquid cooling).

\section{Magnetic Bearings}

Since the inception of our present program we have paid special attention to what we consider to be the key technical problem of the EMB - the magnetic suspension/bearing system. To that end we have initiated a combined theoretical/ experimental program with the objective of developing new classes of magnetic bearings that would be simpler and less expensive than the presently available commercial systems. At this time it is premature to discuss the results of our program, but some comments on the general problem are in order.

As is well known, the first problem that must be faced in designing any magnetic suspension/bearing system is dealing with Earnshaw's Theorem. ${ }^{7}$ This theorem asserts the impossibility of stably levitating an object by means of magnetic forces exerted between elements characterized by constant currents and/or constant magnetization. The theorem implies that if an object is levitated there will be at least one axis in space along which it is unstable. The conventional answers to this dilemma either involve mechanical means of 
stabilization (as in some commercial turbo-vacuum pumps) or the use of sensors and servo circuits to stabilize the bearing system.

Examining the assumptions implicit in the derivation of Earnshaw's Theorem, circumstances can be listed under which it does not apply. For example, it does not apply to diamagnetic bodies, such as superconductors. Indeed magnetic bearings using hightemperature super-conductors have been experimentally tested. 8

Our approaches to simplifying magnetic bearings utilize various combinations of "loopholes" in Earnshaw's Theorem to accomplish stability. Since our presently funded projects are intended for stationary applications, their magnetic bearing systems can be simpler than ones needed for vehicular applications. However, we expect to learn much that will be helpful toward solving the bearing problem for vehicular applications in executing this task.

\section{IV) RESULTS FROM THE TEST PROGRAM}

\section{A. Motor/Generator Performance}

Motor/generator development comprises our work on Halbach arrays and the integration of these arrays with ironless stators. We designed, built, and tested several Halbach arrays using various sizes, materials, and configurations. We found that the measured field strength agreed with predicted values to within several percent. Uniformity of the dipole field was excellent. Magnetic fields outside the arrays were found to be highly self canceling. An assembled generator set produced in excess of $20 \mathrm{~kW}$ at speeds below 20,000 RPM from a single phase winding. Gaps of up to 0.25 " between the magnet array and the stator winding had no discernible effect on motor/generator performance. An AC efficiency of over $92 \%$ was measured for a depth of discharge of $100 \%$. At all speeds of interest, stator impedance was dominated by winding inductance. The high power capability achieved by this system may be attributed to the elimination of iron and resulting low inductance. The high efficiency of the system arises from the elimination of hysteretic loss associated with iron and the low resistance of the stator winding. Harmonic content of the generator output was found to be extremely low: harmonic peaks were more than $50 \mathrm{~dB}$ below fundamental.

\section{B. Power Electronics and Controls}

A number of topologies were explored with the ultimate goal of minimizing total system cost while achieving high efficiency and meeting application specific requirements. Typically, a bridge composed of IGBTs was used to synthesize a variety of waveforms. Waveforms were produced both open loop and by controlling the phase angle between the 
drive waveform and the rotor position. Rotor position was detected with either a sense winding or a Hall sensor. The Hall sensor was preferred for its wide dynamic range. Since our ironless system is inherently insensitive to harmonic content of the drive waveform, modulation of the drive waveform was minimized thus minimizing switching losses. A number of topologies for integrating buck, boost, and modulation functions were developed.

\section{Magnetic Bearings}

The emphasis of the initial phase of our program was on the analytical investigation of magnetic bearing concepts and the development of modeling tools. The modeling tools were developed for a variety of architectures and cover all relevant physical processes including the interaction of magnetic fields produced on the rotor and the stator, supercritical rotor dynamics, etc. Bearing components were built and operated to validate the models. In general, analytical models were found to be valid to within the limits of our ability to measure critical material properties. Components designed with these analytical tools were employed in EMB prototypes.

\section{Separators}

As described above, a critical issue in the design of thick rotor assemblies, necessary for good form factor and efficient energy storage, is the management of the radial variation of centrifugal force. In thick rings, this causes radial tensile stress and large variations in hoop stress through the thickness of the rotor wall. Operation of thick rotors at safe stress levels can entail the inefficient use of expensive material. A well known method for mitigating this problem is the use of multiple materials or layups to achieve a radial gradient in the modulus of elasticity in the hoop direction. While this technique is effective, we believe that employing multiple thinner rings avoids fabrication expense and affords more flexibility in system design. Toward this end, we have designed, analyzed, built and tested a prototype mechanical separator that allows outer rings to grow away from inner rings freely while maintaining sufficient stiffness to avoid flexural resonances in the operating speed range.

\section{E. Containment Systems}

The practical realization of EMB systems requires the containment of energetic fragments in a rotor burst. In our test program to date, we have focused on managing the transfer of energy between fragments and containment structure. Towards this end, we conducted a series of tests in which projectiles made from composite rotor material were fired into containment structures using a two stage gas gun. Velocities in excess of $1 \mathrm{~km} / \mathrm{s}$ were used. We believe that an important function of the containment structure is to trigger the 
disintegration of the rotor in order to mitigate the special hazard of large fragments. We demonstrated containment structures that repeatably caused the composite projectiles to disintegrate into 'cotton candy' or short, threadlike fragments. We also identified containment structures that do not trigger this disintegration. We have applied the lessons learned here to the design of our EMB prototype containment structure.

\section{F. System Integration and Operation}

Two EMB prototypes were built and operated in limited "bench top" tests prior to moving to a test cell. The maximum speed used in this portion of the test program was $42 \%$ of design speed. No composite rotor failures occurred and a number of rotor dynamics issues were identified and resolved. We routinely passed through large amplitude critical frequencies in the range of 4000-6000 RPM to get to the speed range of these tests: from 10,000-25,000 RPM. The units were fairly quiet, producing a faint, pure tone which was only noticeable when ancillary equipment, cooling pumps, vacuum pumps, etc. were turned off. Extraction of power at $20 \mathrm{~kW}$ produced a rapid change in rotor speed and a rapid change in tone. High power operation had no influence on tendencies toward whirl. The most critical issue associated with whirl was found to be management of the relationship between energy dissipated in the rotating frame compared to energy dissipated in the stationary frame. This was addressed through careful bearing design. Throughout the initial test program, stored energy and delivered power were within predicted ranges.

\section{ACKNOWLEDGEMENTS}

The authors would like to acknowledge the contributions of T. Kuklo, G. Dreifurst, $S$ Deteresa, and T.K. Fowler. Work performed under the auspices of the U.S. Department of Energy by the Lawrence Livermore National Laboratory under contract W-7405ENG-48. 


\section{REFERENCES}

1) R.F. Post, T.K. Fowler, and S.F. Post, "A High-Efficiency Electromechanical Battery," Proc. of IEEE., 81 (1993) 462.

2) C.L. Stotler, "Development of Advanced Lightweight Systems Containment Final Report," NASA CR-165212 (May 1981).

3) T.K. Fowler and R.F. Post, "Mechanical Stability of Multiple-Shell Energy Storage Rotors," presented at Western Applied Mechanics Conf., Stanford Research Institute, Sept. 17-19, 1973.

4) E.J. Gunter, Jr., Dynamic Stability of Rotor-Bearing Systems, NASA Sp-113 (1966).

5) R.F. Post and S.F. Post, "Spin Tests of a Multi-Ring Flywheel" (unpublished report).

6) K. Halbach, "Specialty Magnets," Lawrence Berkeley Laboratory Report LBL21945 (July 1986).

7) S. Earnshaw, "On the Nature of the Molecular Forces which Regulate the Constitution of the Luminiferous Ether," Trans. of The Cambridge Philosophical Society, Vol. VII, Part I (1839) 97.

8) B.R. Weinberger, L. Lynds, J.R. Hull, and U. Balachandran, "Low Friction in High Temperature Superconductor Bearings," App. Phs. Lett. 59, August 1991, 1132. 


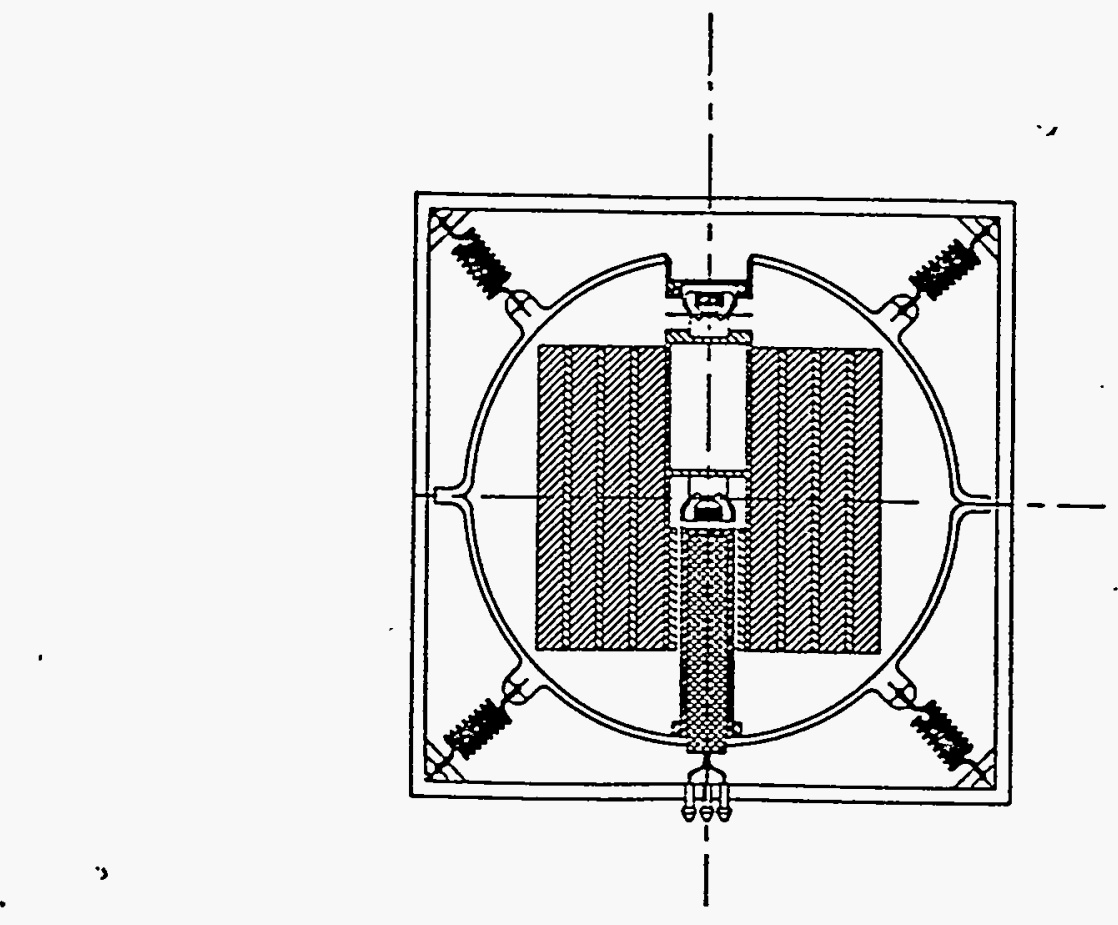

Figure 1. Schematic cutaway drawing of modular EMB. ।

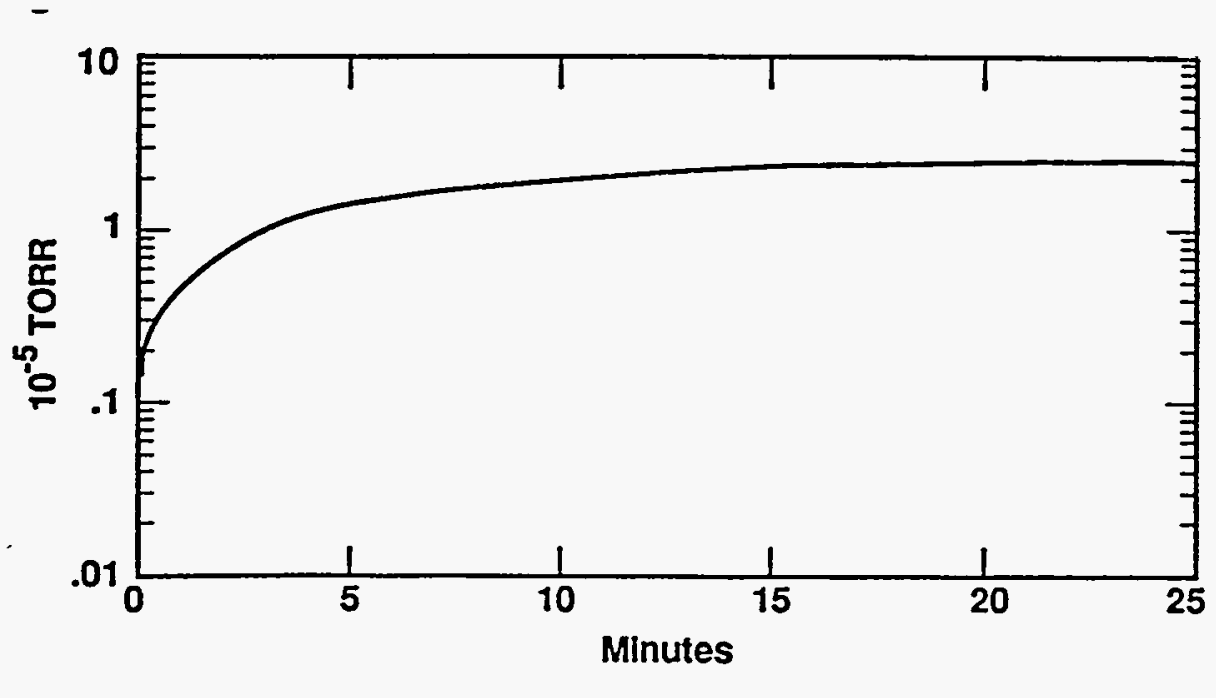

Figure 2. Pressure vs time curve for sealed-off vacuum system containing specially treated epoxy-graphite simulated rotor structure. 


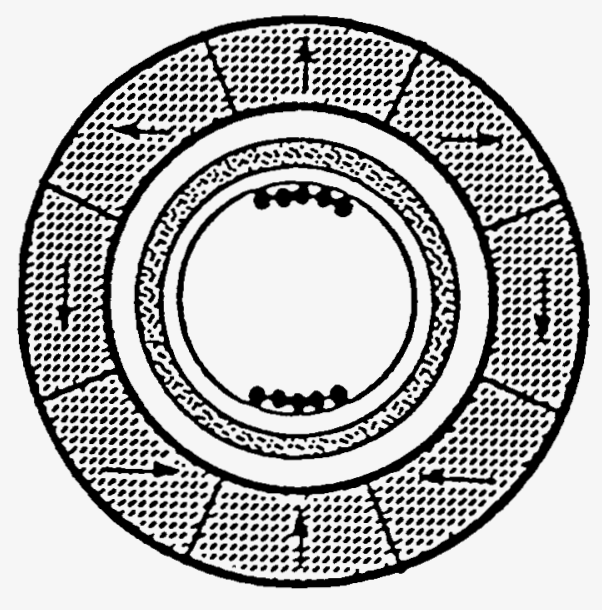

Figure 3. End view of Halbach array showing vacuum barrier and some of windings. 